\section{Reproducibility and diagnostic \\ performance of the vascular index of \\ superb microvascular imaging in real-time \\ breast ultrasonography for evaluating breast masses}

\begin{abstract}
Eun Ji Lee, Yun-Woo Chang, Eunsun Oh, Jiyoung Hwang, Hyun-joo Kim,
\end{abstract} Seong Sook Hong

Department of Radiology, Soonchunhyang University Seoul Hospital, Seoul, Korea

Purpose: This study aimed to evaluate the reproducibility and diagnostic performance of a quantitative parameter of superb microvascular imaging (SMI) in real-time breast ultrasonography (US) for differentiating benign from malignant breast masses.

Methods: Eighty-seven breast masses in 75 patients who underwent both B-mode US and SMI before US-guided core needle biopsy were included in this study. Two radiologists performed B-mode US and measured the vascular index (VI) of SMI respectively for each lesion in real time. Intraobserver and interobserver agreements were analyzed for the VI of SMI. The diagnostic performance of B-mode US using the Breast Imaging Reporting and Database System lexicon and combined use with the VI of SMI was evaluated compared to pathology.

Results: The median VI of malignant masses $(n=32)$ was significantly higher than that of benign masses $(n=55)$ ( $7.6 \%$ and $2.6 \%$, respectively; $P<0.001)$. The intraobserver agreement for VI was excellent regardless of the pathology, size, or depth of the lesion. The interobserver agreement for VI was excellent regardless of the presence of a measurement interval. The interobserver agreement for the final diagnostic decision was improved by combining B-mode US and VI ( $\mathrm{K}=0.883$ ) in comparison with B-mode US only ( $\mathrm{K}=0.617)$. Adding VI led to significant improvements in the specificity $(87.2 \%$ vs. $52.7 \%, 83.6 \%$ vs. $49.0 \%)$, accuracy $(89.7 \%$ vs. $69.3 \%, 84.0 \%$ vs. $65.9 \%$ ) and positive predictive value ( $81.5 \%$ vs. $55.1 \%, 75.6 \%$ vs. $52.6 \%$ ) of B-mode US for both observers compared with B-mode US alone (all, $\mathrm{P}=0.001$ ).

Conclusion: The VI of SMI for real-time breast US is highly reproducible and leads to improved diagnostic performance for differentiating between benign and malignant breast lesions in combination with B-mode US.

Keywords: Breast; Ultrasound, Doppler; Superb microvascular imaging; Neoplasms

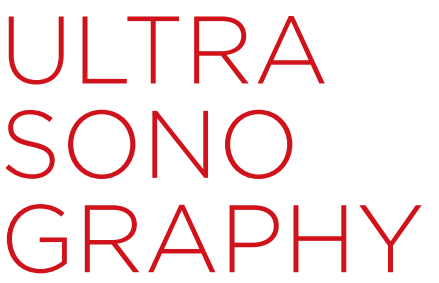

ORIGINAL ARTICLE

https://doi.org/10.14366/usg.20153 pISSN: 2288-5919 - elSSN: 2288-5943 Ultrasonography 2021;40:398-406

Received: September 22, 2020 Revised: November 26, 2020 Accepted: November 27, 2020

Correspondence to: Yun-Woo Chang, MD, Department of Radiology, Soonchunhyang University Seoul Hospital, 59 Daesagwan-ro, Yongsan-gu, Seoul 04401, Korea

Tel. +82-2-709-9066

Fax. +82-2-709-9397

E-mail: ywchang@schmc.ac.kr

This is an Open Access article distributed under the terms of the Creative Commons Attribution NonCommercial License (http://creativecommons.org/ licenses/by-nc/4.0/) which permits unrestricted noncommercial use, distribution, and reproduction in any medium, provided the original work is properly cited.

Copyright (C) 2021 Korean Society of Ultrasound in Medicine (KSUM)



How to cite this article:

Lee EJ, Chang YW, Oh E, Hwang J, Kim HJ, Hong SS. Reproducibility and diagnostic performance of the vascular index of superb microvascular imaging in real-time breast ultrasonography for evaluating breast masses. Ultrasonography. 2021 Jul;40(3):398-406. 


\section{Introduction}

Assessment of the vascularity of a breast mass is important for differentiating benignity from malignancy, as malignant masses show more profound tumor vascularity than benign masses due to neoangiogenesis induced by cancerous cells [1]. Although the Breast Imaging Reporting and Database System (BI-RADS) categorization based on morphology in B-mode ultrasonography (US) takes precedence over other US features in the evaluation of breast masses, tumor vascularity can be noninvasively assessed by Doppler US evaluations of the vascular number and morphology of breast lesions [2-4]. A recent multicenter prospective study showed that the use of color Doppler US in addition to B-mode US could significantly increase specificity in evaluating breast lesions when age was taken into consideration [5]. However, conventional color Doppler US removes clutter caused by tissue motion together with low-speed blood flow signals, thereby limiting the detailed assessment of tumor vascularity through only a qualitative evaluation $[6,7]$.

Superb microvascular imaging (SMI) is an emerging Doppler US technique that can depict slow blood flow apart from clutter using a multi-dimensional filter. By using SMI, it is possible to evaluate the vascularity of a breast mass in greater detail. It has been reported that a qualitative or semi-quantitative evaluation of tumor vascularity using SMI is superior to that of color or power Doppler US, with SMI showing better diagnostic performance in discriminating between malignant and benign breast lesions [812]. A larger number of tumor vessels, central distribution, and branching and penetrating vessels are known to be vascular features associated with malignant breast tumors [8-12]. Microvessels assessed with microvascular Doppler US are also correlated with microvascular density in pathologic specimens, showing stronger correlations than those assessed with color Doppler US $[13,14]$. These results indicate that SMI has advantages in the noninvasive evaluation of vascularity of breast tumors. The introduction of the vascular index (VI) has enabled the quantitative evaluation of tumor vascularity in SMI [15]. A few studies have reported the diagnostic value of $\mathrm{VI}$ in addition to B-mode US for differentiating benign from malignant breast masses $[14,16,17]$. However, those studies focused on the diagnostic performance of $\mathrm{VI}$ in addition to B-mode US without assessing interobserver or intraobserver agreement.

Although it has been reported that the combination of quantitative SMI values with conventional B-mode US can enhance the diagnostic performance in differentiating benign from malignant breast masses, validating the reproducibility of quantitative values is essential for clinical application. In a few previous studies on the diagnostic performance of SMI using VI, the VI of each lesion was retrospectively measured by manually drawing a region of interest (ROI) in a representative image selected using a post-processing program by the operator $[14,16,18]$. No studies have reported the reproducibility or diagnostic performance of VI measured in realtime US performed by different operators.

Thus, the purpose of this study was to evaluate the reproducibility and diagnostic performance of $\mathrm{VI}$ as a quantitative parameter of SMI in real-time breast US and the effects of measurement interval, histopathologic type, and intrinsic factors of lesions on reproducibility.

\section{Materials and Methods}

\section{Patients}

This study involved a retrospective analysis of prospectively acquired data. It was approved by our Institutional Review Board (IRB No. 2020-09-003) and the need to obtain informed consent was waived. From November 2019 to June 2020, both B-mode US and SMI were performed by two radiologists in 128 patients who underwent USguided core needle biopsy for breast lesions on the basis of imaging findings or the physician's decision. Among them, 53 patients were excluded due to the following reasons: prior history of breast cancer with surgery, chemotherapy, or radiotherapy $(n=9)$, prior vacuumassisted biopsy at the biopsy site $(n=2)$, lactating women $(n=1)$, and lesions for which the VI could not be obtained due to the inability to clearly distinguish the ROI, such as a non-mass lesion $(n=44)$. Breast US exams were performed, including B-mode images and SMI, at the time of the biopsy or within 1 month before the biopsy. Finally, 75 patients who had both B-mode US and SMI were included. Twelve patients had two breast masses. A total of 87 breast masses in 75 patients (mean age, 46 years; range, 21 to 83 years) were included in this study.

\section{Real-Time B-Mode Ultrasound Examination}

All US examinations were performed with one of two identical types of US equipment (Aplio 800 system, Cannon Medical Systems Corporation, Tokyo, Japan) using a 7- to $18-\mathrm{MHz}$ multi-frequency ultra-wide band linear array transducer. The examinations were performed by two board-certified radiologists who had 18 and 3 years of experience in breast imaging and had approximately 1 year of experience in SMI, respectively. The US images of B-mode and SMI were obtained for each breast lesion. The operators were aware of patients' clinical information and mammographic findings before the US exams. Two orthogonal B-mode images were obtained for each breast mass and the longest diameter of the lesion was recorded. Lesion depth was recorded as the vertical diameter from the skin to the central portion of the mass and breast thickness was recorded 
as the vertical diameter from the skin to the pectoralis muscle on B-mode images. The ratio of the lesion depth relative to the breast thickness was divided into three equal parts in the total distribution, and the location where the mass was found was classified as superficial, medium, or deep. Each lesion was categorized based on its features on B-mode US according to the American College of Radiology's BI-RADS, 5th edition [19].

\section{Real-Time SMI Ultrasound Examination}

SMI was obtained using the same depth, focus, and time gain as the B-mode images. For the quantitative evaluation of breast masses, the $\mathrm{VI}$ of each lesion was obtained from SMI. For each lesion, the $\mathrm{VI}$ was obtained by manually drawing an $\mathrm{ROI}$ along the margin of the mass in a still image with the maximum Doppler signals during

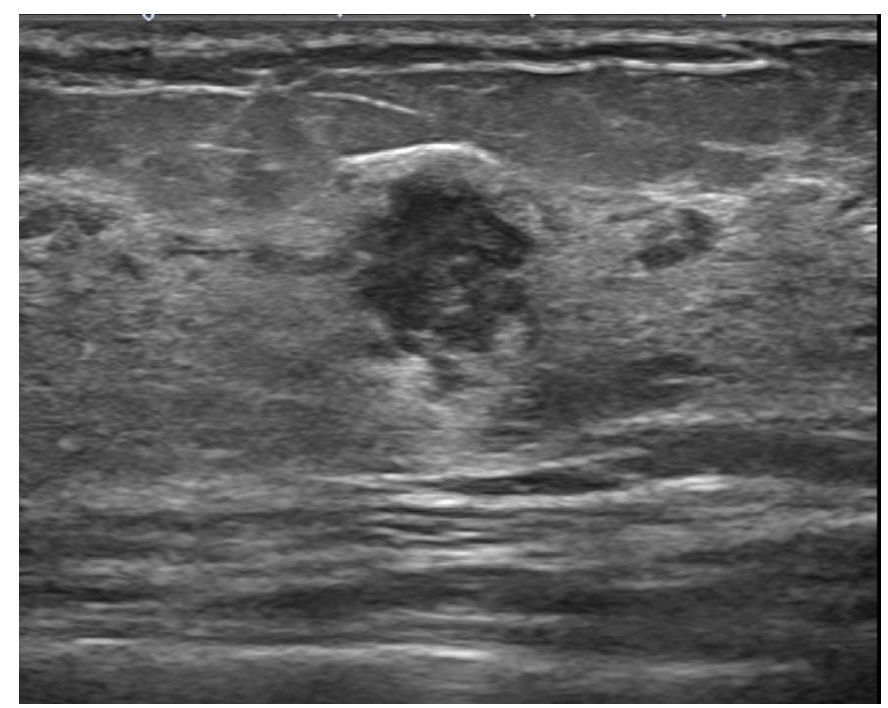

A



a real-time US exam. The $\mathrm{VI}$ is the ratio (expressed as a percentage) between the pixels included within the Doppler signal within the ROI and those of the total lesion. It is automatically calculated using dedicated software provided with the US equipment (Fig. 1). SMI and VI measurements were done at least twice or the same lesion to evaluate intraobserver agreement. If a patient underwent breast US and core needle biopsy on the same day, both observers performed VI measurements on the same day. One observer performed real-time US including B-mode and measurement of the $\mathrm{VI}$ of SMI, followed by the other observer obtaining additional VI measurements for the target lesion in real time. If the patient underwent scheduled core needle biopsy after breast US, the VI measurement was made on a different day by two observers. The $\mathrm{VI}$ of SMI was measured by each observer at different days within



B

Fig. 1. A 49-year-old woman with invasive ductal carcinoma.

A. B-mode ultrasonography (US) shows a 13-mm microlobulated irregular hypoechoic mass at a medium depth in the left breast. $B, C$. Each observer independently measured the vascular index (VI) of superb microvascular imaging (SMI) in real-time by manually drawing a line along the margin of the mass in a still image. The measurement of $\mathrm{VI}$ was obtained by each observer on the same day. The $\mathrm{VI}$ values obtained by the observers were $11.1 \%$ (B, observer 1) and $12.2 \%$ ( $C$, observer 2$)$. Both observers assessed the mass as Breast Imaging Reporting and Database System category 4B based on B-mode US. The VI of SMI obtained by each observer was over the cutoff value of $3.37 \%$. ROI, region of interest. 
1 month of the US exams. If the VI measurement was made on the same day, it was recorded that there was no measurement time interval, and if it was performed on a different day, it was recorded as having a measurement time interval. Each observer performed US exams without knowledge of the images obtained by the other operator. The image parameters for SMI were as follows: velocity scale, $2.5 \mathrm{~cm} / \mathrm{s}$; dynamic range, 21-40 dB; and frame rate, 13-52 frames/s. Data acquisition for the US exams, including B-mode and SMI, took about 2-3 minutes per lesion.

\section{Data and Statistical Analysis}

To compare the size of the lesion and the VI of SMI between benign and malignant masses, the chi-square test or Mann-Whitney $U$ test was used. The BI-RADS assessment categories of lesions based on $B$-mode US were divided into two groups for statistical analysis. Positive results consisted of categories $4 A, 4 B, 4 C$, and 5, while negative results consisted of category 3 . The histopathologic results from core needle biopsy or surgical excision were used as reference standards. If surgical excision was subsequently performed, followed by core needle biopsy, the histopathologic results from the surgical specimen were used.

The interclass correlation coefficient (ICC) was used to assess intraobserver and interobserver agreement for the $\mathrm{VI}$ of SMI [20]. Weighted $\mathrm{k}$ values were used to evaluate interobserver agreements for the BI-RADS category assessment and final category assessment with the combination of B-mode US and the VI of SMI [21]. The $\mathrm{K}$ values were interpreted as indicating poor $(\mathrm{K}=0.0)$, small/slight $(K=0.0-0.20)$, fair $(K=0.21-0.40)$, moderate $(K=0.41-0.60)$, substantial $(\mathrm{K}=0.61-0.80)$, and almost perfect $(\mathrm{K}=0.81-1.00)$ agreement between examiners [21]. Measurement reliability was classified as excellent (ICC $>0.75)$, fair to good (ICC $=0.40-0.75)$, and poor $(I C C \leq 0.40)[22]$. Both ICC scores and $\mathrm{K}$ values were reported with $95 \%$ confidence intervals. Interobserver agreement for $\mathrm{VI}$ according to histopathologic type and intrinsic factors, including lesion size and lesion depth, was also analyzed by calculating the ICC. Interobserver agreement for the final assessment based on B-mode US only and the combination of B-mode US with the VI of SMI of breast masses was assessed using weighted $\mathrm{K}$ values.

The optimal cutoff value was determined based on the average values of the lesion VI measured by two observers. The maximum value of the Youden index in the receiving operating characteristic (ROC) curve analysis was used to obtain the optimal cutoff value of $\mathrm{VI}$. To evaluate the additional diagnostic value of the $\mathrm{VI}$ of SMI to B-mode US, the diagnostic performance based on binary results of positive or negative B-mode US alone and the combined B-mode US and VI of SMI were compared. Sensitivity, specificity, positive predictive value (PPV), negative predictive value (NPV), and accuracy were included as diagnostic performance indices. To evaluate the additional value of the $\mathrm{VI}$ of SMI, we re-assessed BI-RADS category 4A masses by combining the $\mathrm{VI}$ of SMI with B-mode US. If the VI of SMI was less than the cutoff value, downgrading was performed to make the final result negative. The overall diagnostic indices between B-mode US and a combination of B-mode US and the VI of SMI were compared for both observers using the McNemar test. All statistical analyses were performed using Rex 3.1.2 version (https:// rexsoft.org/). P-values less than 0.05 were considered to indicate statistical significance.

\section{Results}

\section{Lesion Characteristics and Histopathologic Diagnoses}

Among a total of 87 lesions, 55 (63.2\%) were diagnosed as benign and 32 (36.8\%) were diagnosed as malignant on US-guided core needle biopsy ( $n=64)$, vacuum-assisted biopsy $(n=6)$, or surgical excision ( $n=17)$. The BI-RADS assessment categories of the lesions based on B-mode US were category $3(n=30)$, category $4 A(n=27)$, category $4 B(n=9)$, category $4 C(n=10)$ and category $5(n=11)$ for observer 1 and category $3(n=29)$, category $4 A(n=26)$, category $4 B$ $(n=17)$, category $4 C(n=6)$, and category $5(n=9)$ for observer 2 . The histopathologic diagnoses of the breast masses are summarized in Table 1. The median size of the malignant masses was significantly $(P=0.022)$ larger than that of benign masses. Malignant masses showed a higher VI than benign masses: $7.3 \%$ (interquartile range [IQR], 3.2\% to $10.3 \%$ ) and $0.9 \%$ (IQR, $0 \%$ to $3.1 \%$ ) for malignant versus benign lesions, respectively $(\mathrm{P}<0.001)$ (Table 1$)$.

Table 1. Comparison of size and vascular index between benign and malignant masses

\begin{tabular}{lllr}
\hline \multicolumn{1}{c}{ Benign $(\mathrm{n}=55)$} & \multicolumn{1}{c}{$\begin{array}{c}\text { Malignant } \\
(\mathrm{n}=32)\end{array}$} & P-value \\
\hline Pathology & $\begin{array}{l}\text { Benign proliferative breast } \\
\text { lesion: } 25,\end{array}$ & IDC: 25, \\
& DCIS: 6, & \\
& fibroadenoma: 21, & ILC with DCIS: 1 & \\
& intraductal papilloma: 3, & & \\
& duct ectasia: 2, & & \\
& sclerosing adenosis: 2, & & \\
& abscess: 1, & & \\
& intramammary lymph & & \\
& node: 1 & & \\
Size (mm) & $9.0(7.0-15.5)$ & $13.5(9.0-21.0)$ & 0.022 \\
Vascular index (\%) & $0.9(0-3.1)$ & $7.3(3.2-10.3)$ & $<0.001$ \\
\hline
\end{tabular}

Values are presented as median (interquartile range).

IDC, invasive ductal carcinoma; DCIS, ductal carcinoma in situ; ILC, invasive lobular carcinoma. 


\section{Intraobserver and Interobserver Agreement of the $\mathrm{VI}$ of SMI in Real-Time US}

Intraobserver agreement for the $\mathrm{VI}$ of SMI was excellent regardless of the pathologic type, size, or depth of the lesion (Table 2). Regarding the pathologic type, the intraobserver agreement was excellent for both benign and malignant lesions (ICC, 0.945 and 0.972 , respectively). When lesions were divided into three groups according to size $(<10 \mathrm{~mm}, 10-20 \mathrm{~mm}$, and $>20 \mathrm{~mm})$, all three groups showed excellent intraobserver agreement (ICC, 0.945, 0.971 , and 0.985 , respectively). The intraobserver agreement was also excellent regardless of whether the lesion depth was superficial, medium, or deep (ICC, 0.975, 0.937, and 0.973). The interobserver agreement was excellent regardless of the presence of a time interval between the $\mathrm{VI}$ measurements (absence of interval: ICC, 0.948; presence of interval: ICC, 0.925) (Table 3, Figs. 1, 2).

There was substantial agreement in the BI-RADS assessment category based on B-mode US ( $\mathrm{K}=0.722)$. The agreement was moderate $(K=0.617)$ when the BI-RADS assessment category was dichotomously divided. When a new dichotomous diagnostic decision was made by applying the VI of SMI to downgrade BI-RADS category 4A lesions, the interobserver agreement was improved, resulting in substantial agreement, with an increase of the $\mathrm{K}$ value $(\mathrm{K}=0.883)$ in comparison with a dichotomous diagnostic decision based on B-mode US alone (Table 3, Fig. 2).

\section{Diagnostic Performances of B-Mode US Alone and Combined B-Mode US with the VI of SMI}

The optimal cutoff value of the VI of SMI was $3.37 \%$, with a

Table 2. Intraobserver agreement of the vascular index according to intrinsic factors of the breast lesions

\begin{tabular}{llll}
\hline & No. (\%) & ICC & $95 \% \mathrm{Cl}$ \\
\hline Pathology & & & \\
Overall & $87(100)$ & 0.960 & $0.935-0.975$ \\
Benign & $55(63.2)$ & 0.945 & $0.879-0.975$ \\
Malignant & $32(36.8)$ & 0.972 & $0.936-0.988$ \\
Size (mm) & & & \\
$<10$ & $37(42.5)$ & 0.945 & $0.879-0.975$ \\
$10-20$ & $20(23.0)$ & 0.971 & $0.935-0.987$ \\
$>20$ & $30(34.5)$ & 0.985 & $0.958-0.995$ \\
Lesion depth ratio ${ }^{\text {a) }}$ & & & \\
Superficial & $26(29.9)$ & 0.975 & $0.944-0.988$ \\
Medium & $35(40.2)$ & 0.937 & $0.876-0.968$ \\
Deep & $26(29.9)$ & 0.973 & $0.940-0.988$ \\
\hline
\end{tabular}

ICC, interclass coefficient; $\mathrm{Cl}$, confidence interval.

${ }^{a}$ The value of the lesion depth divided by the breast thickness. sensitivity of $72.7 \%$, a specificity of $78.1 \%$, and an area under the ROC curve (AUC) of 0.793 . When the optimal cutoff value of $\mathrm{VI}$ was applied to downgrade BI-RADS category 4A lesions, there was an improvement of diagnostic performance for both observers. For observer 1, 21 out of 27 category 4A lesions, and in observer 2, 18 out of 26 category $4 \mathrm{~A}$ lesions were downgraded after applying the optimal cutoff value of VI. In both observers, the Vls of all category 3 lesions were below the cutoff value. In ROC curve analyses, the AUC values were significantly higher for combined use of B-mode US and the VI of SMI than those with B-mode US alone for both observers (observer 1 AUC: 0.748 vs. 0.906, $P<0.001$; observer 2 AUC: 0.715 vs. $0.842, P<0.001$ ) (Fig. 3). The diagnostic indices for $\mathrm{B}$-mode US and the combination of B-mode US and the VI of SMI are summarized in Table 4. For both observers, specificity, PPV, and accuracy were significantly improved by combining the $\mathrm{VI}$ of SMI with B-mode US (specificity from $52.7 \%$ to $87.2 \%$ [P<0.001], PPV from $55.1 \%$ to $81.5 \%[P<0.001]$, and accuracy from $69.3 \%$ to $89.7 \%[P<0.001]$ for observer 1 ; specificity from $49.0 \%$ to $83.6 \%$ $[P<0.001]$, PPV from $52.6 \%$ to $75.6 \%[P=0.001]$, and accuracy from $65.9 \%$ to $84.0 \%$ [P=0.005] for observer 2). For both observers, there were no statistically significant differences in sensitivity or NPV with either imaging method (B-mode US alone or a combination of B-mode US with the VI of SMI).

\section{Discussion}

Our results showed that $\mathrm{VI}$ was a highly reproducible and objective quantitative parameter of SMI. The intraobserver reproducibility of

Table 3. Interobserver agreement of the vascular index of SMI according measurement interval and BI-RADS category

\begin{tabular}{lccc}
\hline & No. (\%) & ICC or kappa & $95 \% \mathrm{Cl}$ \\
\hline $\begin{array}{l}\text { Measurement interval } \\
\text { Absent }{ }^{\text {a) }}\end{array}$ & $57(59.8)$ & $\mathrm{ICC}=0.948$ & $0.910-0.970$ \\
$\begin{array}{l}\text { Present } \\
\text { b) }\end{array}$ & $35(40.2)$ & $\mathrm{ICC}=0.925$ & $0.853-0.962$ \\
$\begin{array}{l}\text { Agreement of } \\
\text { dichotomous division }^{c}\end{array}$ & & & \\
$\begin{array}{l}\text { B-mode } \\
\text { B-mode+VI }\end{array}$ & $87(100)$ & $\mathrm{K}=0.617$ & $0.440-0.794$ \\
\hline Superb (100) & $\mathrm{K}=0.883$ & $0.783-0.984$
\end{tabular}

SMI, superb microvascular imaging; BI-RADS, Breast Imaging Reporting and Database System; ICC, interclass coefficient; $\mathrm{Cl}$, confidence interval; $\mathrm{VI}$, vascular index.

${ }^{\text {a) }}$ Measurement of the $\mathrm{VI}$ on the same day. ${ }^{\mathrm{b})}$ Measurement of the $\mathrm{VI}$ on a different day. 'Dichotomous division: $\mathrm{B}$-mode, benign (BI-RADS category 3), malignant (BIRADS category $4 A, 4 B, 4 C, 5$ ); $B$-mode+ $V I$, downgrade of category $4 B$ lesions according to the cutoff value of the $\mathrm{VI}$. 


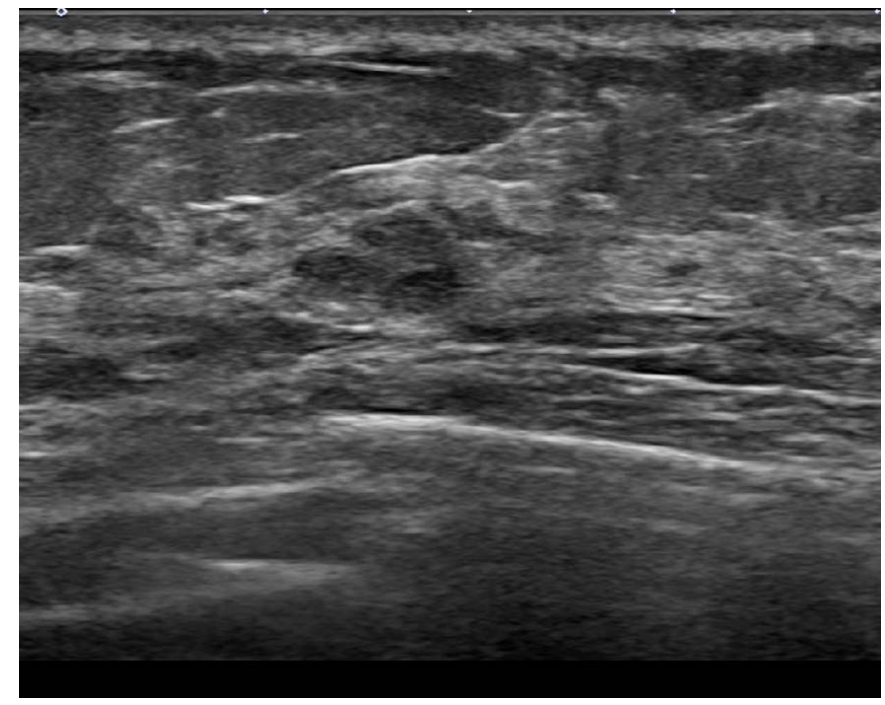

A

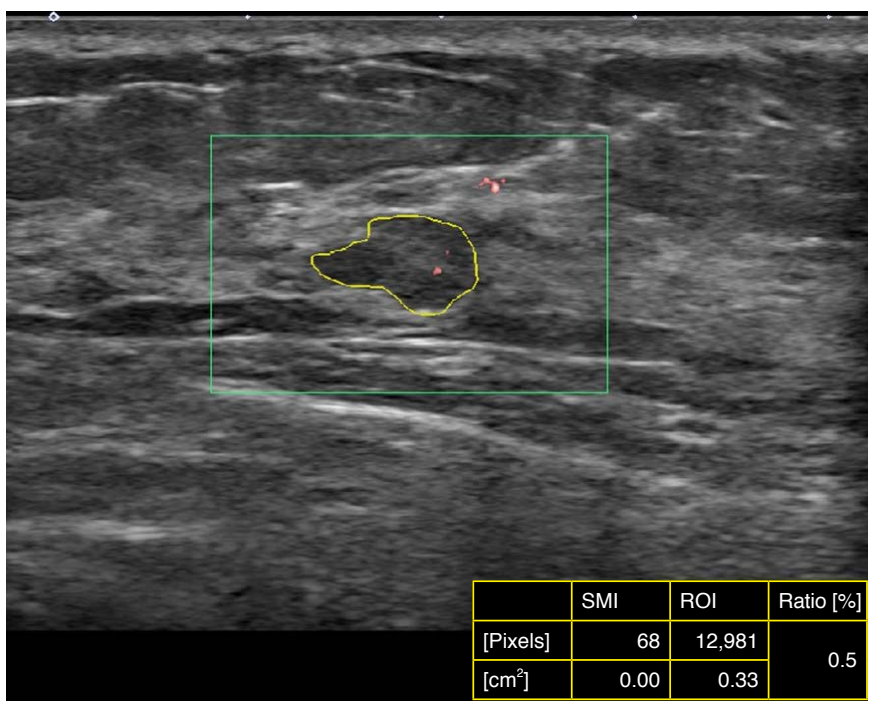

C

VI in real-time US was excellent regardless of the pathology, size, or depth of the lesion.

In our study, the interobserver agreement of $\mathrm{VI}$ in real-time US was excellent for cases with or without a measurement interval between the observers. In VI measurements performed on different days, there might be differences in the ROI placement or SMI settings of the US equipment, including dynamic range or frame rate, which might affect the degree of agreement between examiners. Although the presence of an interval between VI measurements was associated with a mild decrease in the interobserver agreement, the interobserver agreement was still excellent, suggesting high reproducibility of the VI of SMI.

When VI was combined with B-mode US, the diagnostic performance for distinguishing benign and malignant breast masses

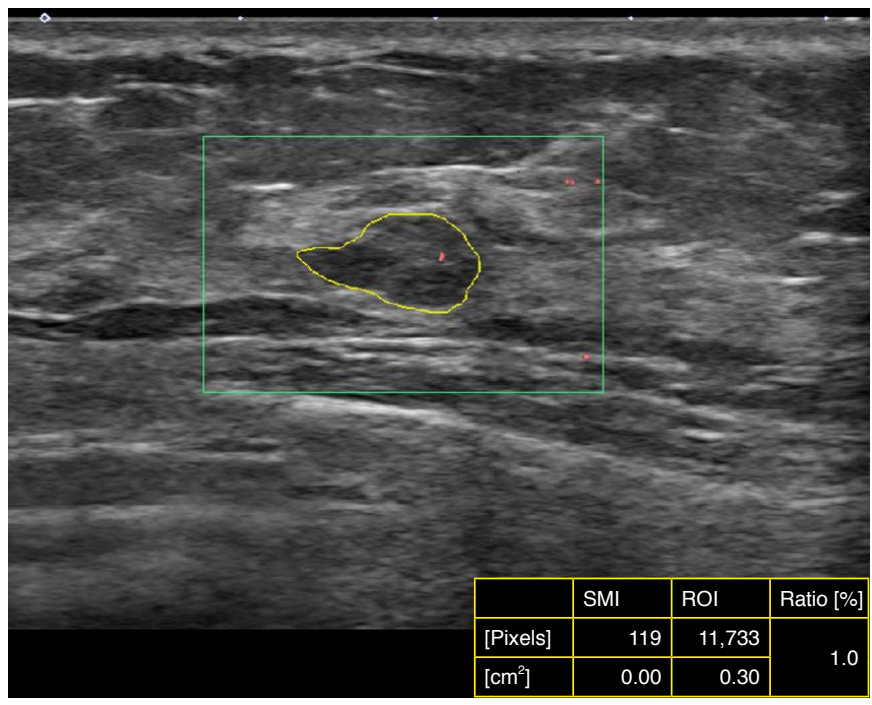

B

Fig. 2. A 37-year-old woman with a left breast mass.

A. B-mode ultrasonography (US) shows an oval, angular, hypoechoic mass in the deep glandular tissue of the left breast. Both observers independently performed B-mode US and superb microvascular imaging (SMI) on different days. The mass was assessed as Breast Imaging Reporting and Database System category $4 \mathrm{~A}$ based on real-time B-mode US by both observers (A). B, C. SMI performed by observer 1 (B) and observer 2 (C) shows minimal Doppler signals within the mass. The vascular index (VI) was calculated as $1.0 \%$ by observer 1 and $0.5 \%$ by observer 2 . As each VI of SMI measured by each observer was less than the cutoff value (3.37\%), the final assessment of combined B-mode US and the VI of SMI was negative for both observers. The mass was pathologically diagnosed as fibroadenoma on core needle biopsy. ROI, region of interest.

improved compared to that with B-mode alone. The interobserver variability in diagnostic decisions also improved. In a previous study, we investigated the value of combining quantitative parameters of shear-wave elastography and SMI with B-mode US and found that adding VI alone to B-mode US could increase the diagnostic performance compared to the use of B-mode alone [17]. In that previous study, VI values measured by different observers were used without analyzing intraobserver or interobserver reproducibility. Chae et al. [18] evaluated interobserver agreement of the VI of SMI and the added value of the VI with B-mode US. They reported that the combination of B-mode US and SMI with VI improved the characterization of breast masses. However, the reader performance was significantly improved for only one reader and they used manually drawn ROls based on image video clips [18]. To the best of 

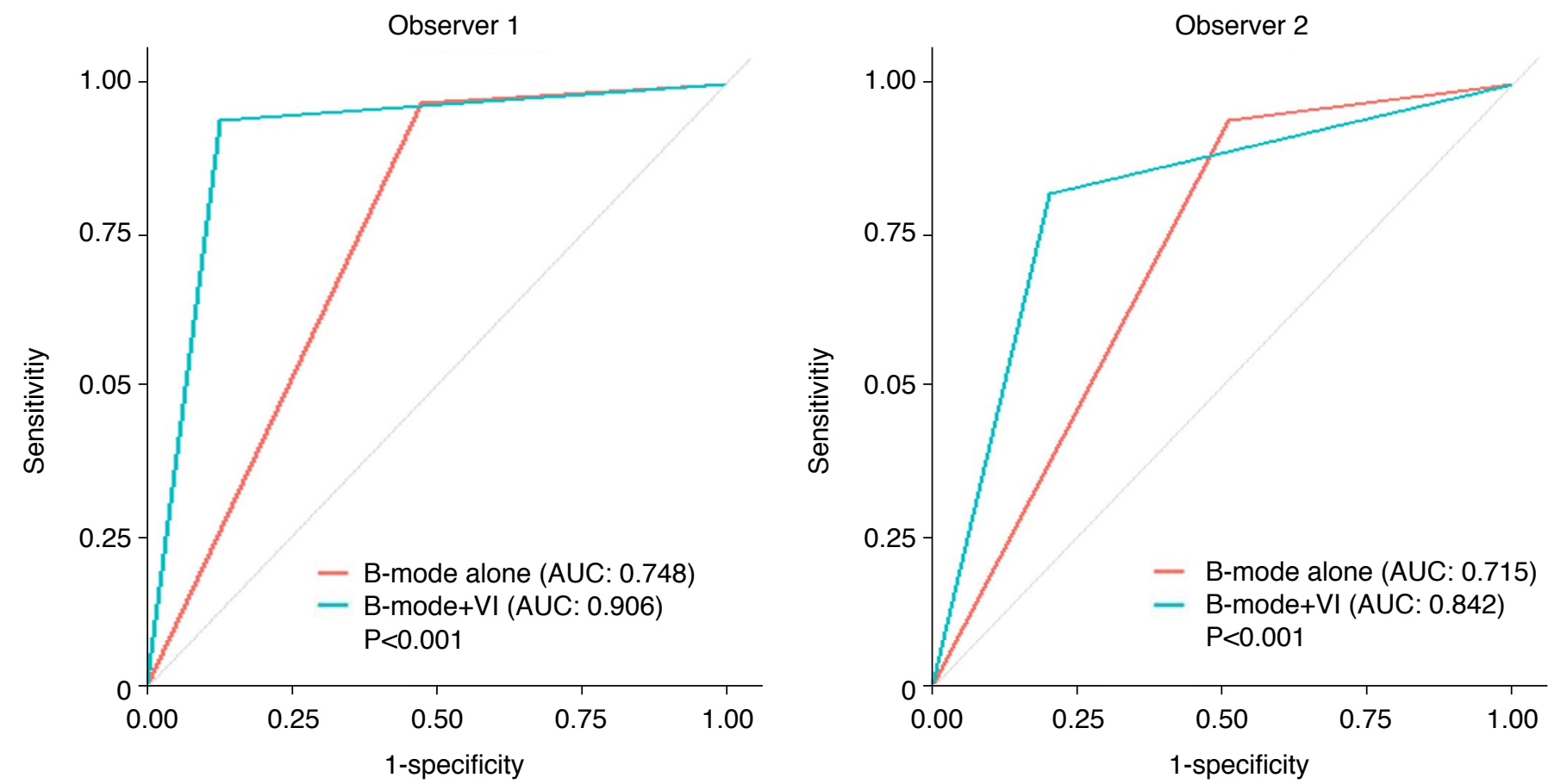

Fig. 3. Receiver operating characteristic curves for B-mode ultrasonography and combined B-mode ultrasonography and vascular index $(\mathrm{VI})$. The area under the curve $(\mathrm{AUC})$ values were significantly higher with the combined use of B-mode ultrasonography and the $\mathrm{VI}$ of superb microvascular imaging than those with B-mode ultrasonography alone for both observers.

Table 4. Diagnostic performance of B-mode US alone and the combination of B-mode US with the VI of SMI

\begin{tabular}{|c|c|c|c|c|c|c|c|c|c|c|}
\hline Variable & Sensitivity (\%) & P-value $e^{a)}$ & Specificity (\%) & P-value ${ }^{a)}$ & Accuracy (\%) & P-value ${ }^{a)}$ & PPV (\%) & P-value ${ }^{a)}$ & NPV (\%) & P-value $e^{a)}$ \\
\hline \multicolumn{11}{|l|}{ Observer 1} \\
\hline B-mode ${ }^{b)}$ & 32/33 (96.9) & 0.343 & 29/55 (52.7) & $<0.001^{\mathrm{a})}$ & $61 / 89(69.3)$ & $<0.001^{\mathrm{a})}$ & $32 / 58(55.1)$ & $<0.001^{\text {a) }}$ & 29/30 (96.6) & 0.833 \\
\hline B-mode+VI & 31/33 (93.9) & & $48 / 55(87.2)$ & & 79/88 (89.7) & & $31 / 38(81.5)$ & & $48 / 50(96.0)$ & \\
\hline \multicolumn{11}{|l|}{ Observer 2} \\
\hline B-mode ${ }^{b)}$ & 31/33 (93.9) & 0.051 & $27 / 55(49.0)$ & $<0.001^{\mathrm{a})}$ & $58 / 88(65.9)$ & $0.005^{\mathrm{a})}$ & $31 / 59(52.6)$ & $0.001^{\mathrm{a})}$ & 27/29 (93.1) & 0.488 \\
\hline B-mode+VI & 28/33 (84.8) & & $46 / 55(83.6)$ & & $74 / 88(84.0)$ & & 28/37 (75.6) & & $46 / 51(90.2)$ & \\
\hline
\end{tabular}

Values are presented as number (\%).

US, ultrasonography; VI, vascular index of SMI; SMI, superb microvascular imaging; PPV, positive predictive value; NPV, negative predictive value; BI-RADS, Breast Imaging Reporting and Database System.

${ }^{\text {a) }}$-values indicate comparison of diagnostic performance of between BI-RADS category alone and the addition of the VI. ${ }^{\text {b) }}$ B-mode: dichotomous division of the BI-RADS assessment category of the lesions based on B-mode ultrasound images; category 3 was assessed as negative and category higher than 4A was assessed as positive, $\mathrm{B}$-mode+VI: downgrading BI-RADS $4 \mathrm{~A}$ masses with the combined use of $\mathrm{VI}$.

our knowledge, our study is the first to evaluate intraobserver and interobserver reproducibility by measuring the real-time $\mathrm{VI}$ of $\mathrm{SMI}$.

The optimal cutoff value obtained in the present study was 3.37\%, similar to the value of $3.35 \%$ found in our previous study [17]. However, different values were reported in previous studies (4\% [16], $8.9 \%$ [14], and 2.95\% [18]). Such differences in the cutoff values of $\mathrm{VI}$ might be due to differences in the measurement methods and learning curve of the performers. Those studies obtained the VI by post-processing software applied to previously obtained images, and the imaging parameters of SMI were somewhat different $[14,17,18]$. One of the reasons for the differences in VI cutoff values among studies may be differences in the study population. Our study included BI-RADS category 3, 4, and 5 lesions, but the highest reported cutoff value of $\mathrm{VI}$ was in a study that enrolled patients who underwent US-guided biopsy for breast lesions classified as BI-RADS category 4 and 5 [14].

The AUC of VI in this study was 0.793 , consistent with the results of previous studies $(0.776$ [16], 0.844 [14], and 0.778 in 
our prior study [17]). To evaluate the value of adding VI to B-mode US, we applied VI to downgrade BI-RADS category 4A lesions. The combination of $\mathrm{VI}$ with B-mode US improved diagnostic performance, showing statistically significant improvements of specificity, PPV, accuracy, and AUC without a significant loss of sensitivity or NPV for both observers. These results are consistent with previous studies using the $\mathrm{VI}$ of SMI $[14,17,18]$, suggesting that applying $\mathrm{VI}$ for downgrading category $4 \mathrm{~A}$ lesions can reduce unnecessary biopsies.

We also evaluated the effect of the combined use of VI with B-mode US on interobserver variability in the final diagnostic decision. The interobserver agreement of the dichotomous division of the final diagnostic decision based on B-mode alone was improved after adding the VI of SMI to B-mode US. Considering that observers in this study had different experiences in breast US, these results meant that the addition of SMI could decrease the variability of diagnostic decision between observers with different expertise in B-mode US.

The present study has a few limitations. First, this study included a limited number of patients and had a retrospective design. Thus, it might have been affected by selection bias. In addition, all included patients were scheduled for biopsies. The assessments of their lesions might have been overrated, which might have lowered the specificity. Second, the VI value did not reflect the overall vascularity of the lesion. Since the measurements of $\mathrm{VI}$ were obtained by selecting a two-dimensional still image of SMI in real-time and drawing an ROI manually, it was impossible to quantify the total volumetric vascularity of the lesion. This will be possible with the development of technology that enables quantification of three-dimensional volume data from SMI. Third, the two observers had about 1 year of accumulated experience of VI measurements with a similar learning curve, which might have resulted in high interobserver agreement. Lastly, the time interval of VI measurements was varied within 1 month, which could have led to bias between the two observers. For generalized use of this quantitative parameter of microvascular Doppler US, further studies using various techniques of microvascular Doppler US including a larger number of patients are warranted.

In conclusion, the VI of SMI obtained in real-time breast US showed substantial intraobserver and interobserver agreement regardless of the intrinsic factors of the lesion or the presence of a measurement interval. Adding the VI of SMI to conventional B-mode US improved diagnostic performance for differentiating benign from malignant breast lesions. It also improved the interobserver agreement of the final diagnostic decision. Thus, VI, as a highly reproducible quantitative parameter of SMI, might provide additional objective information useful for distinguishing benign from malignant breast masses.

ORCID: Eun Ji Lee: https://orcid.org/0000-0002-4860-2495; Yun-Woo Chang: https:// orcid.org/0000-0001-9704-8112; Eunsun Oh: https://orcid.org/0000-0001-5160390X; Jiyoung Hwang: https://orcid.org/0000-0002-3070-4880; Hyun-joo Kim: https://orcid.org/0000-0001-5386-1881; Seong Sook Hong: https://orcid.org/00000003-2893-6583

\section{Author Contributions}

Conceptualization: Chang YW. Data acquisition: Lee EJ, Chang YW. Data analysis or interpretation: Lee EJ, Chang YW. Drafting of the manuscript: Lee EJ, Chang YW. Critical revision of the manuscript: Chang YW, Oh E, Hwang J, Kim HJ, Hong SS. Approval of the final version of the manuscript: all authors.

\section{Conflict of Interest}

No potential conflict of interest relevant to this article was reported.

\section{Acknowledgments}

This research was supported by the Soonchunhyang University research fund.

\section{References}

1. Yadav L, Puri N, Rastogi V, Satpute P, Sharma V. Tumour angiogenesis and angiogenic inhibitors: a review. J Clin Diagn Res 2015;9:XE01-XE05.

2. Lee SW, Choi HY, Baek SY, Lim SM. Role of color and power doppler imaging in differentiating between malignant and benign solid breast masses. J Clin Ultrasound 2002;30:459-464.

3. Kook SH, Park HW, Lee YR, Lee YU, Pae WK, Park YL. Evaluation of solid breast lesions with power Doppler sonography. J Clin Ultrasound 1999;27:231-237.

4. Giuseppetti GM, Baldassarre S, Marconi E. Color Doppler sonography. Eur J Radiol 1998;27 Suppl 2:S254-S258.

5. Watanabe T, Kaoku S, Yamaguchi T, Izumori A, Konno S, Okuno T, et al. Multicenter prospective study of color Doppler ultrasound for breast masses: utility of our color Doppler method. Ultrasound Med Biol 2019;45:1367-1379.

6. Lee WJ, Chu JS, Houng SJ, Chung MF, Wang SM, Chen KM. Breast cancer angiogenesis: a quantitative morphologic and Doppler imaging study. Ann Surg Oncol 1995;2:246-251.

7. Yang WT, Tse GM, Lam PK, Metreweli C, Chang J. Correlation between color power Doppler sonographic measurement of breast tumor vasculature and immunohistochemical analysis of microvessel density for the quantitation of angiogenesis. J Ultrasound Med 2002;21:1227-1235.

8. Ma Y, Li G, Li J, Ren WD. The diagnostic value of superb microvascular imaging (SMI) in detecting blood flow signals of 
breast lesions: a preliminary study comparing SMI to color Doppler flow imaging. Medicine (Baltimore) 2015;94:e1502.

9. Park AY, Seo BK, Woo OH, Jung KS, Cho KR, Park EK, et al. The utility of ultrasound superb microvascular imaging for evaluation of breast tumour vascularity: comparison with colour and power Doppler imaging regarding diagnostic performance. Clin Radiol 2018;73:304-311.

10. Park AY, Seo BK, Cha SH, Yeom SK, Lee SW, Chung HH. An innovative ultrasound technique for evaluation of tumor vascularity in breast cancers: superb micro-vascular imaging. J Breast Cancer 2016;19:210-213.

11. Yongfeng Z, Ping Z, Wengang L, Yang S, Shuangming T. Application of a novel microvascular imaging technique in breast lesion evaluation. Ultrasound Med Biol 2016;42:2097-2105.

12. Zhan J, Diao XH, Jin JM, Chen L, Chen Y. Superb microvascular imaging: a new vascular detecting ultrasonographic technique for avascular breast masses: a preliminary study. Eur J Radiol 2016;85:915-921.

13. Ma Y, Li J, Ren W, Deng L. Correlation between superb microvascular imaging and pathological microvessel density in breast tumors. J Xiangya Med 2016;1:30.

14. Park AY, Kwon M, Woo OH, Cho KR, Park EK, Cha SH, et al. A prospective study on the value of ultrasound microflow assessment to distinguish malignant from benign solid breast masses: association between ultrasound parameters and histologic microvessel densities. Korean J Radiol 2019;20:759-772.

15. Park AY, Seo BK. Up-to-date Doppler techniques for breast tumor vascularity: superb microvascular imaging and contrast-enhanced ultrasound. Ultrasonography 2018;37:98-106.

16. Zhang XY, Zhang L, Li N, Zhu QL, Li JC, Sun Q, et al. Vascular index measured by smart 3-D superb microvascular imaging can help to differentiate malignant and benign breast lesion. Cancer Manag Res 2019;11:5481-5487.

17. Lee EJ, Chang YW. Combination of quantitative parameters of shear wave elastography and superb microvascular imaging to evaluate breast masses. Korean J Radiol 2020;21:1045-1054.

18. Chae EY, Yoon GY, Cha JH, Shin HJ, Choi WJ, Kim HH. Added value of the vascular index on superb microvascular imaging for the evaluation of breast masses: comparison with grayscale ultrasound. J Ultrasound Med 2021;40:715-723.

19. Mendelson EB, Bohm-Velez M, Berg WA, Whitman GJ, Feldman MI, Madjar $\mathrm{H}$, et al. ACR BI-RADS ultrasound. In: D'Orsi CJ, Sickles EA, Mendelson EB, Morris EA, eds. ACR BI-RADS Atlas, Breast Imaging Reporting and Data System. 5th ed. Reston, VA: American College of Radiology, 2013;1-173.

20. Stanish WM, Taylor N. Estimation of the intraclass correlation coefficient for the analysis of covariance model. Am Stat 1983;37:221-224.

21. Cohen J. Weighted kappa: nominal scale agreement with provision for scaled disagreement or partial credit. Psychol Bull 1968;70:213220.

22. Hudson JM, Milot L, Parry C, Williams R, Burns PN. Inter- and intraoperator reliability and repeatability of shear wave elastography in the liver: a study in healthy volunteers. Ultrasound Med Biol 2013;39:950-955. 